\title{
Acoustokinetics: Crafting force landscapes from sound waves
}

\author{
Mohammed A. Abdelaziz $\left[\right.$ and David G. Grier ${ }^{\circ}$ \\ Department of Physics and Center for Soft Matter Research, New York University, New York, New York 10003, USA
}

(Received 8 October 2019; revised manuscript received 11 November 2019; accepted 16 January 2020; published 19 February 2020)

\begin{abstract}
Factoring the pressure field of a harmonic sound wave into its amplitude and phase profiles provides the foundation for an analytical framework for studying acoustic forces that not only provides insights into the forces exerted by specified sound waves but also addresses the inverse problem of designing sound waves to implement desired force landscapes. We illustrate the benefits of this acoustokinetic framework through case studies of purely nonconservative force fields, standing waves, pseudostanding waves, and tractor beams.
\end{abstract}

DOI: 10.1103/PhysRevResearch.2.013172

\section{INTRODUCTION}

Structured sound waves exert forces and torques that can be harnessed to transport, sort, and organize insonated objects [1-4]. Applications include noncontact processing of sensitive [5,6] and hazardous [7] materials, flow focusing for materials analysis and medical diagnostics [8], and automated remote manipulation for research [9]. Rapidly growing interest in harnessing acoustic forces has inspired a fundamental reassessment of the physics of wave-mediated forces. Recent developments in the theory of acoustic forces [10-14] parallel the analogous theory of optical forces $[15,16]$. Both offer valuable and often surprising insights into the elementary mechanisms of wave-matter interactions. The acoustokinetic framework introduced here addresses the complementary inverse problem: identifying what wave will create a desired force landscape.

The inverse problem for optical forces recently has been rendered more tractable by expressing the electromagnetic field in terms of its real-valued amplitudes and phases along each Cartesian coordinate [17,18]. This approach is called the theory of photokinetic effects and yields useful analytic expressions for the performance of optical traps [19] including design criteria for optical tractor beams [18]. Here, we show that an analogous factorization of the pressure field in sound waves is similarly useful for understanding and implementing acoustic manipulation. We illustrate the utility of this acoustokinetic framework through case studies on nonconservative acoustic force fields, standing and pseudostanding waves, and acoustic tractor beams.

\section{A. Light: Photokinetic analysis}

We develop acoustokinetics by analogy to photokinetics and therefore briefly review the theory of optical forces. A

Published by the American Physical Society under the terms of the Creative Commons Attribution 4.0 International license. Further distribution of this work must maintain attribution to the author(s) and the published article's title, journal citation, and DOI. small particle immersed in an electromagnetic wave develops an electric dipole moment proportional to the local field. This induced dipole experiences a time-averaged Lorentz force in gradients of the field that can be expressed as [16]

$$
\mathbf{F}_{e}(\mathbf{r})=\frac{1}{2} \operatorname{Re}\left\{\alpha_{e} \sum_{j=1}^{3} E_{j}(\mathbf{r}) \nabla E_{j}^{*}(\mathbf{r})\right\},
$$

where $E_{j}(\mathbf{r})$ is the $j$ th Cartesian coordinate of the electric field and $\alpha_{e}$ is the particle's complex dipole polarizability. Expressing the components of the electric field in terms of their real-valued amplitude and phase profiles,

$$
E_{j}(\mathbf{r})=u_{j}(\mathbf{r}) e^{i \varphi_{j}(\mathbf{r})},
$$

yields the surprisingly simple expression [17],

$$
\mathbf{F}_{e}(\mathbf{r})=\frac{1}{4} \alpha_{e}^{\prime} \nabla \sum_{j=1}^{3} u_{j}^{2}(\mathbf{r})+\frac{1}{2} \alpha_{e}^{\prime \prime} \sum_{j=1}^{3} u_{j}^{2}(\mathbf{r}) \nabla \varphi_{j}(\mathbf{r}),
$$

where $\alpha_{e}^{\prime}$ and $\alpha_{e}^{\prime \prime}$ are the real and imaginary parts of the polarizability, respectively.

The first term on the right-hand side of Eq. (3) is the manifestly conservative intensity-gradient force responsible for single-beam optical traps such as optical tweezers [20]. The second describes a nonconservative force [21] that is directed by phase gradients [22]. Phase-gradient forces tend to drive trapped particles out of thermodynamic equilibrium with their supporting media [22,23], mediate the transfer of light's orbital angular momentum [24-27], and have been used to create light-driven micromachines such as pumps [28], mixers [29], and optical tractor beams [30]. Even nonabsorbing dielectric particles experience nonconservative optical forces because of radiative contributions to the dipole polarizability $[31,32]$.

The dipole-order expression in Eq. (3) accurately describes the forces experienced by particles with radii, $a_{p}$, that are small enough to satisfy the Rayleigh criterion, $k a_{p}<$ 1 , where $k$ is the wave number of light. In the Rayleigh regime, the conservative intensity-gradient force generally dominates the light-matter interaction because $\alpha_{e}^{\prime}$ scales as $\left(k a_{p}\right)^{3}$, whereas $\alpha_{e}^{\prime \prime}$ scales as $\left(k a_{p}\right)^{6}$. 


\section{B. Sound: Acoustic radiation forces}

The analogous dipole-order acoustic radiation force experienced by a small particle in a harmonic sound field may be expressed in terms of the pressure, $p(\mathbf{r}, t)$, as [14]

$$
\mathbf{F}(\mathbf{r})=\frac{1}{2} \operatorname{Re}\left\{\alpha_{a} p \nabla p^{*}+\beta_{a} k^{-2}(\nabla p \cdot \nabla) \nabla p^{*}\right\},
$$

where the coefficients $\alpha_{a}$ and $\beta_{a}$ play the role of dipole and quadrupole polarizabilities, respectively. This expression is analogous to Eq. (1) for optical forces and is obtained by rearranging terms from Eq. (16) in Ref. [14]. It therefore also is equivalent [14] to the angular spectrum decomposition of $\mathbf{F}(\mathbf{r})$ [33] for $k a_{p}<1$. Expressing $\mathbf{F}(\mathbf{r})$ in terms of multipole polarizabilities clarifies the analogy with photokinetics. Lengths in Eq. (4) are scaled by the wave number, $k=\omega / c_{m}$, where $\omega$ is the sound's frequency and $c_{m}$ is its speed in the medium. Equation (4) applies to inviscid fluids, for which the pressure satisfies the scalar wave equation

$$
\nabla^{2} p=-k^{2} p .
$$

Our focus on traveling waves in inviscid media is inspired by our interest in developing modalities of long-ranged noncontact manipulation. Long-range manipulation is facilitated by minimizing acoustic losses in the medium. This can be achieved in air by working at frequencies below $50 \mathrm{kHz}[3,34]$, for which the acoustic attenuation is less than $2 \mathrm{~dB} \mathrm{~m}^{-1}$ under standard conditions [35] and scales as $\omega^{2}$ for lower frequencies. The equivalent limiting frequency for water is roughly $2 \mathrm{MHz}$ [36]. Working at low frequencies also minimizes the influence of acoustic streaming forces, which ordinarily compete with acoustic radiation forces in viscous media and in inviscid media bounded by confining surfaces [37].

An object's dipole and quadrupole polarizabilities generally depend on its size, shape, and composition as well as the frequency of the sound and the properties of the fluid medium. For simplicity and concreteness, we will specialize to the case of a spherical scatterer of radius $a_{p}$ that is composed of a material of density $\rho_{p}$ and sound speed $c_{p}$. Such an object's response to the sound field is characterized by the polarizabilities [14]

$$
\begin{aligned}
\alpha_{a} & =\frac{4 \pi a_{p}^{3}}{3 \rho_{m} c_{m}^{2}} f_{0}\left[-1+i \frac{1}{3}\left(f_{0}+f_{1}\right)\left(k a_{p}\right)^{3}\right], \\
\beta_{a} & =\frac{2 \pi a_{p}^{3}}{\rho_{m} c_{m}^{2}} f_{1}\left[1+i \frac{1}{6} f_{1}\left(k a_{p}\right)^{3}\right],
\end{aligned}
$$

where the monopole coupling coefficient,

$$
f_{0}=1-\frac{\rho_{m} c_{m}^{2}}{\rho_{p} c_{p}^{2}}
$$

depends on the compressibility mismatch between the particle and the medium, and the dipole coupling coefficient,

$$
f_{1}=2 \frac{\rho_{p}-\rho_{m}}{2 \rho_{p}+\rho_{m}},
$$

gauges the density mismatch. These expressions also are obtained by reorganizing coefficients from Eq. (16) of Ref. [14] and are valid for $k a_{p}<1$. They constitute the leading-order

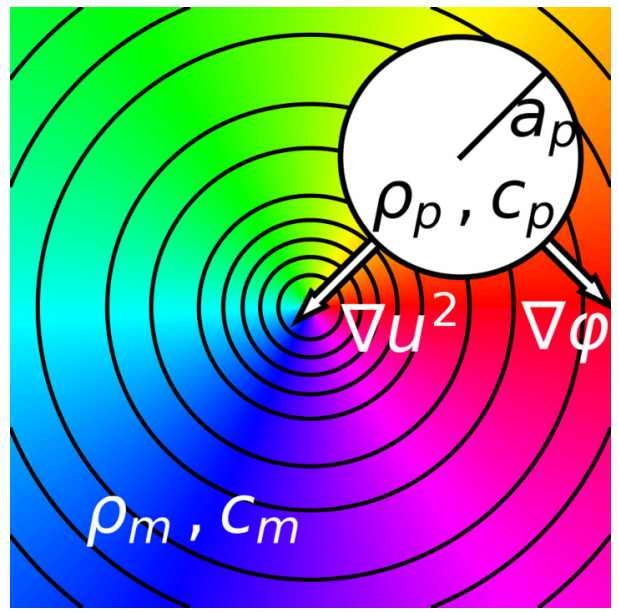

FIG. 1. Schematic representation of a sphere of radius $a_{p}$ immersed in an acoustic pressure field. Contours denote isosurfaces of the pressure intensity. Colors represent the phase of the pressure field. Generally speaking, intensity gradients direct conservative trapping forces while phase gradients direct nonconservative driving forces.

contributions for both the real parts of the polarizabilities, $\alpha_{a}^{\prime}$ and $\beta_{a}^{\prime}$, and also the imaginary parts, $\alpha_{a}^{\prime \prime}$ and $\beta_{a}^{\prime \prime}$.

\section{ACOUSTOKINETIC FRAMEWORK}

Drawing on the analogy with photokinetics, we express the harmonic sound wave's pressure field in terms of its amplitude and phase profiles:

$$
p(\mathbf{r}, t)=u(\mathbf{r}) e^{i \varphi(\mathbf{r})} e^{-i \omega t} .
$$

The first term on the right-hand side of Eq. (4) then yields

$$
\mathbf{F}_{\alpha}(\mathbf{r})=\frac{1}{4} \alpha_{a}^{\prime} \nabla u^{2}+\frac{1}{2} \alpha_{a}^{\prime \prime} u^{2} \nabla \varphi,
$$

which is directly analogous to Eq. (3) for the dipole-order force exerted by light. These contributions to the acoustic radiation force are depicted in Fig. 1. As in the optical case, $\alpha_{a}^{\prime}$ and $\alpha_{a}^{\prime \prime}$ scale as $\left(k a_{p}\right)^{3}$ and $\left(k a_{p}\right)^{6}$ respectively, which means that the conservative force generally dominates for small particles.

The second term on the right-hand side of Eq. (4) arises from the velocity-matching condition at the sphere's boundary and so has no analog in optical radiation forces. It vanishes for density-matched particles $\left(\beta_{\alpha}=0\right)$, which therefore behave exactly like dielectric particles in a light field, in agreement with conclusions from previous studies [38]. When expressed in terms of the amplitude and phase profiles, this term separates naturally into a conservative contribution,

$$
\mathbf{F}_{\beta}^{c}(\mathbf{r})=\frac{1}{4} \beta_{a}^{\prime} \nabla\left(u^{2}+\frac{1}{2} k^{-2} \nabla^{2} u^{2}\right),
$$

that augments the conservative intensity-gradient force from $\mathbf{F}_{\alpha}(\mathbf{r})$ and a nonconservative contribution,

$$
\begin{aligned}
\mathbf{F}_{\beta}^{n c}(\mathbf{r})= & \frac{1}{4} \beta_{a}^{\prime \prime} k^{-2}\left[\left(2 k^{2} u^{2}+\nabla^{2} u^{2}+2 u \nabla u \cdot \nabla\right) \nabla \varphi\right. \\
& \left.-\left(u \nabla^{2} \varphi+2 u \nabla \varphi \cdot \nabla\right) \nabla u\right],
\end{aligned}
$$


that is directed both by phase gradients and also by amplitude gradients. The combination,

$$
\mathbf{F}_{\beta}(\mathbf{r})=\mathbf{F}_{\beta}^{c}(\mathbf{r})+\mathbf{F}_{\beta}^{n c}(\mathbf{r}),
$$

captures the sphere's leading-order coupling to the quadrupole components of the incident field. A derivation of Eq. (9) from Eq. (4) is presented in the Appendix.

Unlike the optical case, where quadrupolar forces generally are weaker than dipole contributions, the two terms in $\mathbf{F}_{\beta}(\mathbf{r})$ can be comparable in magnitude to their counterparts in $\mathbf{F}_{\alpha}(\mathbf{r})$ because $\beta_{a}^{\prime}$ scales as $\left(k a_{p}\right)^{3}$ and $\beta_{a}^{\prime \prime}$ scales as $\left(k a_{p}\right)^{6}$. These density-dependent terms therefore can be used to exert control in ways that are not possible with light.

For very small particles satisfying $k a_{p} \ll 1$, the acoustic force field is dominated by the conservative terms proportional to $\alpha_{a}^{\prime}$ and $\beta_{a}^{\prime}$. These terms are identical to the force described by the classic Gor'kov potential [12], which is widely used to describe acoustic trapping phenomena $[3,39]$. For larger particles, and for appropriately structured sound fields, nonconservative contributions proportional to $\alpha_{a}^{\prime \prime}$ and $\beta_{a}^{\prime \prime}$ can be significant, and even can be dominant $[40,41]$. Such contributions are not accounted for by the Gor'kov potential.

The acoustokinetic framework described by Eq. (9) is the principal contribution of this work. We now demonstrate its value through case studies on realizable sound fields with exceptional properties.

\section{APPLICATIONS OF THE ACOUSTOKINETIC FRAMEWORK}

\section{A. Designing purely nonconservative force fields}

To illustrate how the acoustokinetic framework can address the inverse problem of designing sound waves to implement desired force landscapes, we use Eq. (9) to design harmonic sound waves that exert purely nonconservative forces. This is equivalent to requiring the conservative part of the acoustic radiation force to vanish and thus requires us to look beyond the Gor'kov potential. Equations (9a) and (9b) show that this goal can be met if the particle is not density matched, $\beta_{a}^{\prime} \neq 0$, and if the pressure intensity satisfies the inhomogeneous Helmholtz equation,

$$
\nabla^{2} u^{2}+2\left(1+\frac{\alpha_{a}^{\prime}}{\beta_{a}^{\prime}}\right) k^{2} u^{2}=C .
$$

The undetermined constant $C$ distinguishes families of nonconservative sound waves for the class of objects with compatible values of $\alpha_{a}^{\prime} / \beta_{a}^{\prime}$. Solutions to Eq. (10) must be real-valued and must be paired with real-valued phase profiles that complete the description of the pressure field and satisfy the wave equation, Eq. (5).

One interesting set of purely nonconservative solutions has the sinusoidal amplitude profile

$$
u(\mathbf{r})=p_{0} \cos (q(x-y)),
$$

with spatial frequency

$$
q=\frac{1}{2} \sqrt{1+\frac{\alpha_{a}^{\prime}}{\beta_{a}^{\prime}}} k
$$

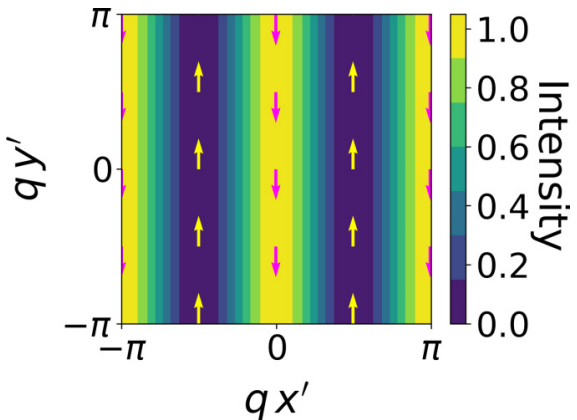

FIG. 2. Intensity of the "picket fence" field in the $\left(x^{\prime}, y^{\prime}\right)$ plane. For particularly selected parameters, the conservative force vanishes everywhere in this field, and the remaining force (indicated by arrows) is purely nonconservative. With the choice of material properties plotted $\left(C_{2} / C_{1}=1 / 3\right)$, the direction of the force is also spatially modulated. The sign of the force in the $\hat{y}^{\prime}$ direction is also its sign in the $\hat{z}$ direction.

The associated phase profile,

$$
\varphi(\mathbf{r})=k z \cos \gamma+(x+y) \sqrt{\frac{1}{2} k^{2} \sin ^{2} \gamma-q^{2}},
$$

identifies this field as the superposition of two plane waves, each oriented at angle $\gamma$ relative to $\hat{z}$ and at angle

$$
\theta=\cos ^{-1}\left(-\frac{\alpha_{a}^{\prime}}{\beta_{a}^{\prime}}\right)
$$

relative to one another in the $(x, y)$ plane. Under these conditions, the in-plane component of the radiation pressure exactly cancels the conservative intensity-gradient force. The remaining scattering force is sinusoidally modulated in the transverse plane. The result is a "picket fence" of parallel force lines, which is illustrated in Fig. 2 using the rotated coordinates

$$
\begin{aligned}
& x^{\prime}=x-y \quad \text { and } \\
& y^{\prime}=x+y
\end{aligned}
$$

for clarity. In these coordinates, the net force,

$$
\mathbf{F}\left(\mathbf{r}^{\prime}\right)=\frac{1}{4} k p_{0}^{2} f\left(x^{\prime}\right) \hat{F},
$$

is directed along

$$
\hat{F}=\sqrt{\frac{1}{2} \sin ^{2} \gamma-\frac{q^{2}}{k^{2}}} \hat{y}^{\prime}+\cos \gamma \hat{z}
$$

and has an amplitude that varies with the transverse coordinate as

$$
f\left(x^{\prime}\right)=C_{1}+2\left(C_{2}-C_{1}\right) \cos ^{2}\left(q x^{\prime}\right) .
$$

The scale and depth of the force landscape's modulation depend on the object's properties through $C_{1}=\beta_{a}^{\prime \prime}\left(1+\alpha_{a}^{\prime} / \beta_{a}^{\prime}\right)$ and $C_{2}=\alpha_{a}^{\prime \prime}+\beta_{a}^{\prime \prime}$.

Picket fence modes for a given type of object are distinguished by the angle of inclination, $\gamma$. The range of possible angles is limited by Eq. (11c) to

$$
\sin ^{2} \gamma>\frac{2 q^{2}}{k^{2}} \text {. }
$$


With this constraint, picket fences can be created for objects that satisfy

$$
-1<\frac{\alpha_{a}^{\prime}}{\beta_{a}^{\prime}}<\sin ^{2} \gamma .
$$

Under some conditions, including those depicted in Fig. 2, the direction of the force can alternate within the fringe pattern. In terms of the standard coupling coefficients, alternating picket fences can be projected for objects satisfying

$$
-\frac{3}{4}<\frac{f_{0}}{f_{1}}<-\frac{1}{2}
$$

Droplets of xylene hexafluoride $\left(\rho_{p}=1370 \mathrm{~kg} \mathrm{~m}^{-1}, c_{p}=\right.$ $\left.880 \mathrm{~m} \mathrm{~s}^{-1}\right)$ dispersed in butanol $\left(\rho_{m}=810 \mathrm{~kg} \mathrm{~m}^{-3}, c_{m}=\right.$ $1240 \mathrm{~m} \mathrm{~s}^{-1}$ ), for example, have $f_{0} / f_{1}=-0.55$ and thus are predicted to experience an alternating picket fence force field. Although alternating picket fences are only possible for a limited domain of material properties, picket fences in general could have practical applications for sorting objects by density or compressibility.

\section{B. Conservative forces in standing waves}

The acoustokinetic framework also is useful for analyzing the force fields created by specified sound waves. Standing waves, for example, can be decomposed into superpositions of counterpropagating plane waves. The phase-dependent terms in $\mathbf{F}_{\alpha}(\mathbf{r})$ and $\mathbf{F}_{\beta}(\mathbf{r})$ vanish in such superpositions, leaving a manifestly conservative force landscape,

$$
\mathbf{F}_{\text {standing }}(\mathbf{r})=\frac{1}{4} \nabla\left[\alpha_{a}^{\prime} u^{2}+\beta_{a}^{\prime} k^{-2}(\nabla u)^{2}\right] .
$$

Particles satisfying

$$
\alpha_{a}^{\prime}-\beta_{a}^{\prime}>0
$$

are drawn toward antinodes of the pressure field, as illustrated in Fig. 3. This condition is satisfied by compressible, lowdensity particles such as bubbles in water. Particles with complementary properties are drawn toward nodes.

\section{Nonconservative forces in pseudostanding waves}

Not all zero-momentum waves are standing waves. Some have nontrivial phase profiles and so can exert nonconservative forces. The archetype for such pseudostanding waves is a superposition of three plane waves with equal amplitude, $p_{0} / 3$, and wave vectors

$$
\mathbf{k}_{n}=-k\left[\cos \left(n \frac{2 \pi}{3}\right) \hat{x}+\sin \left(n \frac{2 \pi}{3}\right) \hat{y}\right]
$$

that satisfy $\sum_{n=1}^{3} \mathbf{k}_{n}=0$ [42]. The pressure intensity for such a three-wave superposition is plotted in Fig. 4(a) and displays a sixfold rotational symmetry similar to that of the corresponding sixfold standing wave in Fig. 3(b). The triangular lattice of antinodes in Fig. 4(a), however, is meshed with a dual hexagonal lattice of nodes, as indicated by dotted circles in Fig. 4(a). Because nonconservative forces tend to be weaker than conservative forces by a factor of $\left(k a_{p}\right)^{3}$, we focus our attention on regions where the sound field forms stable traps, and expand about these points in polar coordinates, $\mathbf{r}=(r, \theta)$ for small displacements, $k r<1$.
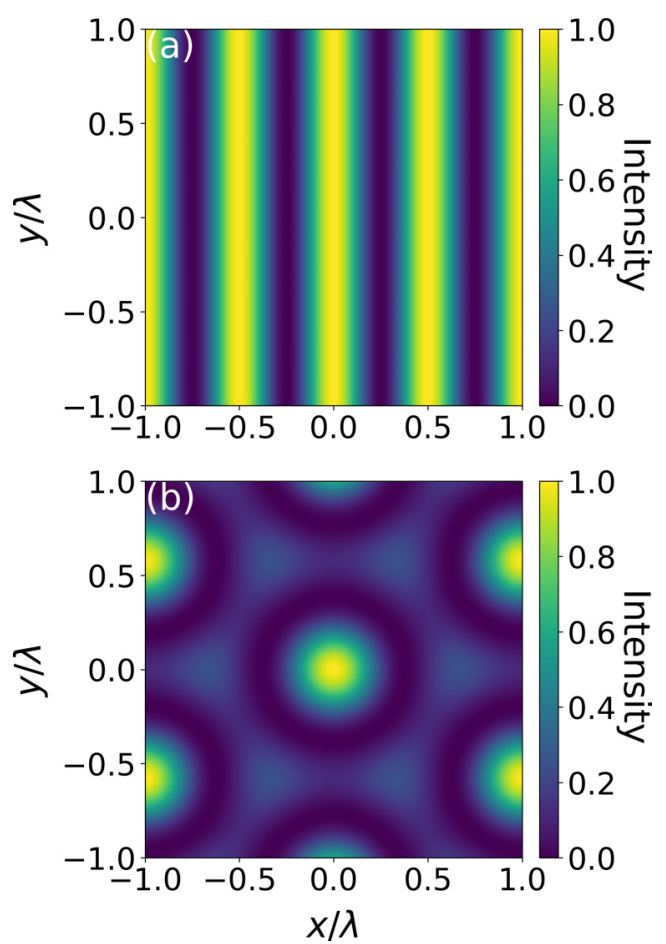

FIG. 3. Maps of the pressure intensity of (a) a one-dimensional standing wave and (b) a sixfold standing wave. Particles are trapped either at intensity maxima or minima, depending on their composition relative to the medium.

For the threefold pseudostanding wave, the conservative contributions from Eqs. (9a) and (9b) simplify to

$$
\mathbf{F}^{c}(\mathbf{r})=\frac{1}{8}\left(2 \alpha_{a}^{\prime}-\beta_{a}^{\prime}\right) \nabla u^{2} .
$$

Particles satisfying

$$
2 \alpha_{a}^{\prime}-\beta_{a}^{\prime}>0
$$

therefore are drawn to pressure antinodes while complementary particles seek out nodes.

A node-seeking particle experiences amplitude and phase profiles of the form

$$
\begin{aligned}
& u_{\text {node }}(\mathbf{r}) \approx \frac{1}{2} p_{0} k r \quad \text { and } \\
& \varphi_{\text {node }}(\mathbf{r}) \approx \pm \theta-\frac{\pi}{2} .
\end{aligned}
$$

The conservative part of the associated force field exerts a Hookean restoring force,

$$
\mathbf{F}_{\text {node }}^{c}(\mathbf{r}) \approx-\frac{1}{16} k p_{0}^{2}\left(\beta_{a}^{\prime}-2 \alpha_{a}^{\prime}\right) k r \hat{r}
$$

that keeps the particle localized near the node. The force field also includes a nonconservative component,

$$
\mathbf{F}_{\text {node }}^{n c}(\mathbf{r}) \approx \pm \frac{1}{8} k p_{0}^{2}\left(\alpha_{a}^{\prime \prime}+\beta_{a}^{\prime \prime}\right) k r \hat{\theta},
$$

that is directed by the azimuthal phase gradient and causes the displaced particle to orbit its node. The pseudostanding wave therefore transfers orbital angular momentum to particles moving near its nodes, with each node acting as a unit-charge acoustic vortex [1]. The sign of the orbital angular momentum alternates from site to site on the honeycomb lattice of nodes. 

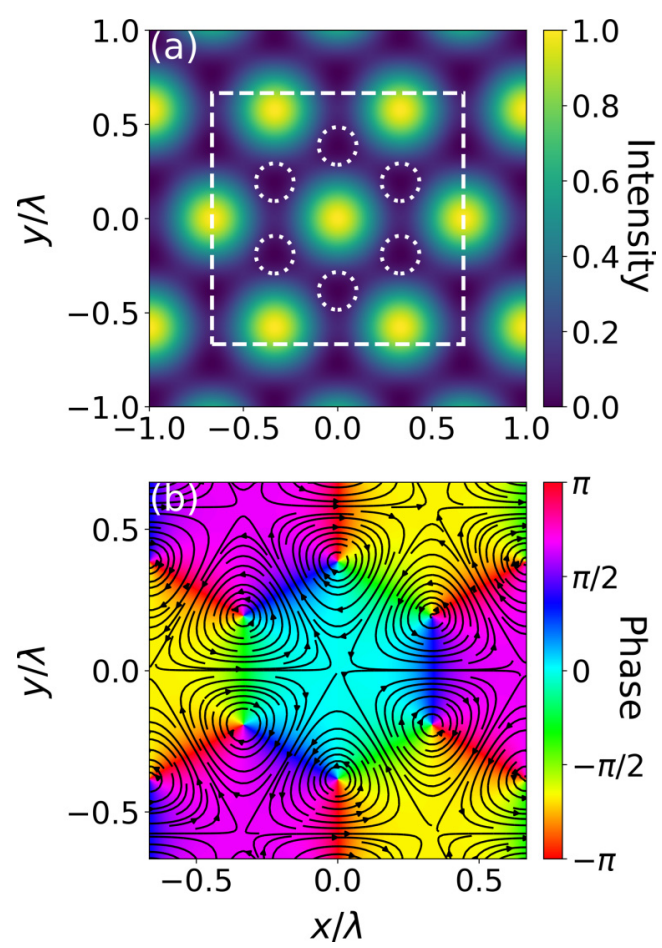

FIG. 4. (a) Intensity and (b) phase maps of the threefold zeromomentum wave. Dashed white circles indicate the intensity nodes and phase singularities in the field and the dashed white square indicates the region plotted in panel (b). Streamlines show the direction of the phase gradient, along which nonconservative forces act.

The array of alternating acoustic vortices in a pseudostanding wave therefore carries no net angular momentum [43].

Nodes repel particles satisfying $2 \alpha_{a}^{\prime}>\beta_{a}^{\prime}$, which instead seek out the triangular lattice of antinodes. Near an antinode, the sound field's amplitude and phase profiles are

$$
\begin{aligned}
& u_{\text {antinode }}(\mathbf{r}) \approx p_{0}\left[1-\frac{1}{4}(k r)^{2}\right] \text { and } \\
& \varphi_{\text {antinode }}(\mathbf{r}) \approx \frac{1}{24}(k r)^{3} \cos 3 \theta .
\end{aligned}
$$

For small displacements, the conservative terms from $\mathbf{F}_{\alpha}(\mathbf{r})$ and $\mathbf{F}_{\beta}(\mathbf{r})$ exert a Hookean restoring force on an antinodeseeking particle:

$$
\mathbf{F}_{\text {antinode }}^{c}(\mathbf{r})=-\frac{1}{8} k p_{0}^{2}\left(2 \alpha_{a}^{\prime}-\beta_{a}^{\prime}\right) k r \hat{r} .
$$

The nonconservative terms create a sextupole flow that tends to drive the particle from one antinode to another:

$$
\mathbf{F}_{\text {antinode }}^{n c}(\mathbf{r})=F_{0} k^{2} r^{2}(\cos 3 \theta \hat{r}-\sin 3 \theta \hat{\theta}),
$$

where $F_{0}=k p_{0}^{2}\left(2 \alpha_{a}^{\prime \prime}-\beta_{a}^{\prime \prime}\right) / 32$.

The conservative part of the pseudostanding wave's force field vanishes for materials satisfying $2 \alpha_{a}^{\prime}=\beta_{a}^{\prime}$, leaving a purely nonconservative force field. This contrasts with the force exerted by a standing wave, which is always conservative. Realizing this condition in practice, however, would require a precise balance of material properties.

More generally, systems satisfying $2 \alpha_{a}^{\prime \prime}-\beta_{a}^{\prime \prime}>2 \alpha_{a}^{\prime}-\beta_{a}^{\prime}$ will experience nonconservative forces that rival conservative trapping forces. In the particular case of an air bubble of size $k a_{p}=0.3$ in water, for example, the nonconservative force exceeds the conservative force for displacements greater than $k r \approx 0.025$ from the pressure antinodes. A pseudostanding wave at amplitude $p_{0}=1 \mathrm{kPa}$ and frequency $f=\omega /(2 \pi)=$ $2 \mathrm{MHz}$ yields a nonconservative force on the order of $1 \mathrm{nN}$ for a displacement of $k r=0.1$ and a conservative force of just $0.1 \mathrm{nN}$. The overall force field, being mostly nonconservative, resembles the streamlines in Fig. 4.

These observations illustrate that nonconservative forces can emerge along directions where the sound field carries no net momentum. More generally, it shows that radiation pressure can be directed independently of the direction of wave propagation. This independence can be used to craft tractor beams from propagation-invariant Bessel beams [18,30,44].

\section{Bessel beams and tractor beams}

Both the standing wave and the pseudostanding wave require boundary conditions that completely enclose their targets. Long-range manipulation without physical confinement is best achieved with propagation-invariant traveling waves. The natural basis for such applications is the family of Bessel beams [44-46], which are nondiffracting solutions to Eq. (5) in cylindrical coordinates, $\mathbf{r}=(r, \theta, z)$. The amplitude and phase profiles for a Bessel beam propagating along $\hat{z}$ are

$$
\begin{aligned}
& u_{\gamma, n}(\mathbf{r})=p_{0} J_{n}(k r \sin \gamma) \quad \text { and } \\
& \varphi_{\gamma, n}(\mathbf{r})=k z \cos \gamma+n \theta,
\end{aligned}
$$

respectively, where $J_{n}(\cdot)$ is a Bessel function of the first kind of order $n$. Bessel beams are distinguished by the convergence angle $\gamma$ that ranges from $\gamma=0$ for conventional plane waves to $\gamma=\pi / 2$ for circular standing waves and the integer $n$ that imposes a helical pitch on the beam's wavefronts.

The conservative part of a Bessel beam's force field is directed radially. Beams with $n=0$ have maximum intensity along the axis, $r=0$. Those with $n>0$ have zero intensity on the axis. The radial component of the acoustic force is linear in $k r$ for $|n| \leqslant 2$ and scales as $(k r)^{3}$, or higher, for $|n|>2$. Whether the force attracts the particle to the axis or repels it depends on the particle's properties. For simplicity, we restrict our analysis to $|n| \leqslant 2$, in which case the conditions for particles to be trapped on axis are

$$
\begin{aligned}
4 \alpha_{a}^{\prime}+\beta_{a}^{\prime}(1+3 \cos 2 \gamma) & >0, & & n=0, \\
\alpha_{a}^{\prime}+\beta_{a}^{\prime} \cos 2 \gamma & <0, & & n= \pm 1, \\
\beta_{a}^{\prime} & <0, & & n= \pm 2 .
\end{aligned}
$$

As noted recently [47], the condition for stable trapping by an $n=0$ Bessel beam differs qualitatively from the analogous condition in Eq. (18) for trapping at an antinode of a standing wave. For example, dense objects with large values of $\beta_{a}^{\prime}$ are repelled by the antinodes of standing waves and pseudostanding waves, but tend to be trapped by the central antinode of a Bessel beam with $\gamma<\pi / 4$. Similar reversals arise for trapping at the central node of Bessel beams with $|n|>0$.

Having established the condition for stable transverse trapping, we next analyze the axial force on a particle localized at $r=0$. Any beam satisfying $\left.\mathbf{F}(\mathbf{r})\right|_{r=0} \cdot \hat{z}<0$ can be said to act as a tractor beam. It should be noted that optical Bessel 
beams do not act as tractor beams for small objects because the dipole-order photokinetic force is always repulsive [18].

Axial forces in propagation-invariant Bessel beams are inherently nonconservative. The relevant terms in Eq. (9) yield

$$
\begin{aligned}
\left.\mathbf{F}(\mathbf{r})\right|_{n=0}, & =\frac{1}{2}\left[\left(\alpha_{a}^{\prime \prime}+\beta_{a}^{\prime \prime}\right) u^{2}+\frac{\beta_{a}^{\prime \prime}}{2 k^{2}} \nabla^{2} u^{2}\right] \nabla \varphi \\
& =\frac{1}{2} p_{0}^{2} k\left(\alpha_{a}^{\prime \prime}+\beta_{a}^{\prime \prime} \cos ^{2} \gamma\right) \cos \gamma \hat{z} .
\end{aligned}
$$

Unlike optical Bessel beams, therefore, acoustic Bessel beams can act as tractor beams for particles satisfying both the trapping condition from Eq. (26a) and also

$$
\alpha_{a}^{\prime \prime}+\beta_{a}^{\prime \prime} \cos ^{2} \gamma<0
$$

Expressed in terms of material properties, these conditions simplify to

$$
\begin{aligned}
& f_{0}<\frac{3}{8}(1+3 \cos 2 \gamma) f_{1} \text { and } \\
& \left(\frac{f_{0}}{f_{1}}\right)^{2}+\frac{f_{0}}{f_{1}}+\frac{3}{4} \cos ^{2} \gamma<0 .
\end{aligned}
$$

Figure 5 shows the domain of beam shapes and particle compositions for which the $n=0$ Bessel beam acts as a tractor beam. These include the optimal condition $f_{0} / f_{1}=-1 / 2$ that was identified in the original discussion of acoustic tractor beams [44].

Density-matched objects $\left(f_{1}=0\right)$ can only be trapped if they are compressible enough that $f_{0}<0$. This means, however, that $\mathbf{F} \cdot \hat{z}>0$, from which we conclude that Bessel beams are not tractor beams for such objects. This is reflected in Fig. 5(b), which presents the axial pulling force as a function of the relative sound speed, $c_{m} / c_{p}$, and density, $\rho_{m} / \rho_{p}$ for a strongly converging Bessel beam with $\gamma=70^{\circ}$. For this convergence angle, the previously discussed system of xylene hexafluoride droplets dispersed in butanol will experience a tractor force in the Rayleigh regime.

The analogous treatment for $n=1$ yields

$$
\begin{aligned}
\left.\mathbf{F}(\mathbf{r})\right|_{n=1} & =\frac{\beta_{a}^{\prime \prime}}{4 k^{2}} \nabla^{2} u^{2} \nabla \varphi \\
& =\frac{1}{4} p_{0}^{2} k \beta_{a}^{\prime \prime} \cos \gamma \sin ^{2} \gamma \hat{z} .
\end{aligned}
$$

Because $\beta_{a}^{\prime \prime}>0$, such beams do act as tractor beams for any choice of materials, at least not for objects trapped on the axis. They instead drive trapped objects downstream.

The $n=2$ beam can trap small objects on the axis, but exerts no axial force at all,

$$
\left.\mathbf{F}(\mathbf{r})\right|_{n=2}=0 \text {. }
$$

Such beams might serve as useful conduits for Rayleigh particles that are moved back and forth along the axis by other forces.

\section{DISCUSSION}

The theory of acoustokinetic forces presented here expresses the influence of a sound wave on a small object in

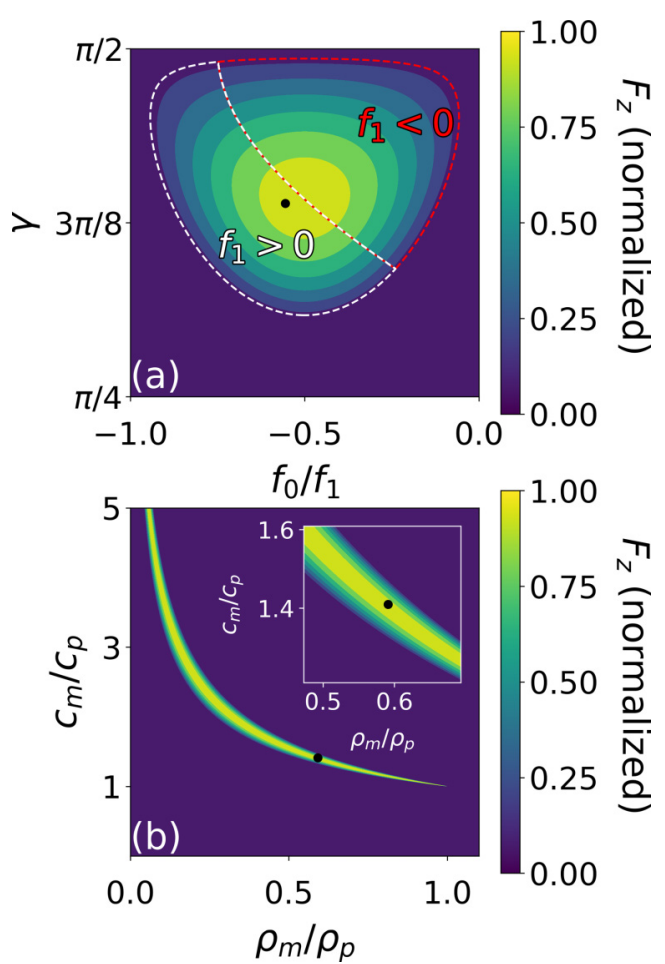

FIG. 5. Relative strength of the axial force, $\left.F_{z} \equiv \mathbf{F}(\mathbf{r})\right|_{r=0} \cdot \hat{z}$, acting on an object that is trapped along the axis of an $n=0$ Bessel beam. A value of 0 indicates that the object is not trapped on axis. (a) Dense objects with $f_{1}>0$ are pulled upstream by the Bessel beam under conditions enclosed by the white curve and are repelled under complementary conditions enclosed by the red curve. These conditions are reversed for buoyant objects with $f_{1}<0$. Densitymatched objects are always repelled. The Bessel beam acts like a tractor beam for all other objects along the interface between the red- and white-bordered regions. (b) The axial pulling force as a function of material parameters for the specific case $\gamma=70^{\circ}$. The black point indicates parameters for droplets of the industrial solvent xylene hexafluoride in butanol. Inset: the region near this system.

terms of the amplitude and phase profiles of the pressure field. The resulting analytical framework, which is summarized in Eq. (9), offers a complementary perspective on acoustic forces to the standard development, which explicitly refers to the velocity field. Acoustokinetic expressions are particularly useful for designing acoustic force fields because they inherently account for coupling between the pressure and velocity fields and specify amplitude and phase profiles that can be controlled with transmissive, reflective, or emissive elements.

The acoustokinetic approach yields expressions that closely resemble results for optical forces in the Rayleigh limit. Exploring the differences and similarities between acoustokinetic and photokinetic forces offers insights into how sound fields couple to material properties to generate useful and interesting force landscapes. The most important difference is that the particle's polarizability includes contributions from the incident wave's monopole component. This means that the force arising from the sound field's dipole component includes terms at the same order of magnitude as leading quadrupole contributions. Such mixing does not arise in optical forces. It vanishes for objects that are density 
matched with the medium, yielding expressions for acoustic forces that are exactly analogous to their optical counterparts.

The acoustokinetic framework naturally accounts for the conservative nature of the force field exerted by standing waves and differentiates it from the influence of pseudostanding waves that also carry no net momentum yet still exert nonconservative forces. When applied to acoustic Bessel beams, the acoustokinetic framework provides clear guidance on the limits for axial trapping and the conditions under which a zeroth-order Bessel beam can act as a tractor beam. This result contrasts with the equivalent analysis of optical Bessel beams, which do not act as tractor beams in the Rayleigh regime.

These examples illustrate the value of the acoustokinetic framework for analyzing acoustic force fields. More generally, the expressions from Eq. (9) can be solved either numerically, or in some cases analytically, for the phase and amplitude profiles that correspond to a specified force field acting on a particle of a given size, compressibility, and density. As in the optical case, the framework can be extended beyond the dipole approximation. For objects substantially smaller than the wavelength of sound, however, the dipole-order terms are simple enough to yield analytical expressions. This framework also can be extended to handle interactions among insonated objects, an effect usually referred to as secondary Bjerknes forces.

\section{ACKNOWLEDGMENTS}

This work was supported by the MRSEC program of the National Science Foundation through Award No. DMR1420073. The authors acknowledge helpful conversations with Phillip Marston (Washington State University) and Konstantin Bliokh (The Australian National University and RIKEN, Japan).

\section{APPENDIX: DERIVATION OF EQ. (9)}

The central idea of the acoustokinetic framework is to factor the incident pressure field $p(\mathbf{r})$ into its real-valued amplitude $u(\mathbf{r})$ and phase $\varphi(\mathbf{r})$ :

$$
p(\mathbf{r})=u(\mathbf{r}) e^{i \varphi(\mathbf{r})} .
$$

Expressed in these terms, the Helmholtz wave equation, Eq. (10), separates into two coupled differential equations that constrain the amplitude and phase profiles,

$$
\begin{aligned}
\nabla^{2} u+k^{2} u-u(\nabla \varphi)^{2} & =0, \\
u \nabla^{2} \varphi+2 \nabla u \cdot \nabla \varphi & =0 .
\end{aligned}
$$

These constraints transform the expression for the timeaveraged acoustic force in Eq. (4) into the acoustokinetic expressions in Eq. (9).

The right-hand side of Eq. (4) is readily expanded into the sum of four terms,

$$
\begin{aligned}
\mathbf{F}(\mathbf{r})= & \frac{1}{2}\left[\alpha_{a}^{\prime} \operatorname{Re}\left\{p \nabla p^{*}\right\}-\alpha_{a}^{\prime \prime} \operatorname{Im}\left\{p \nabla p^{*}\right\}\right. \\
& +k^{-2} \beta_{a}^{\prime} \operatorname{Re}\left\{(\nabla p \cdot \nabla) \nabla p^{*}\right\} \\
& \left.-k^{-2} \beta_{a}^{\prime \prime} \operatorname{Im}\left\{(\nabla p \cdot \nabla) \nabla p^{*}\right\}\right],
\end{aligned}
$$

each of will be expressed in terms of $u(\mathbf{r})$ and $\varphi(\mathbf{r})$. Substituting Eq. (A1) into Eq. (A3a) and noting that

$$
p \nabla p^{*}=\frac{1}{2} \nabla u^{2}-i u^{2} \nabla \varphi
$$

leads directly to Eq. (9a). The first term on the right-hand side of Eq. (A4) is the gradient of an analytic function and therefore corresponds to a manifestly conservative force. We demonstrate that the second term corresponds to a purely nonconservative force by showing that it is divergence free:

$$
\begin{aligned}
\nabla \cdot\left(u^{2} \nabla \varphi\right) & =\nabla\left(u^{2}\right) \cdot \nabla \varphi+u^{2} \nabla^{2} \varphi \\
& =2 u \nabla u \cdot \nabla \varphi+u^{2} \nabla^{2} \varphi \\
& =0,
\end{aligned}
$$

with the last line following from Eq. (A2b).

The expressions in Eqs. (A3b) and (A3c) depend on the real and imaginary parts of $(\nabla p \cdot \nabla) \nabla p^{*}$. The real part is conveniently transformed using the vector identity,

$$
\begin{aligned}
\nabla(\mathbf{A} \cdot \mathbf{B})= & (\mathbf{A} \cdot \boldsymbol{\nabla}) \mathbf{B}+(\mathbf{B} \cdot \boldsymbol{\nabla}) \mathbf{A} \\
& +\mathbf{A} \times(\boldsymbol{\nabla} \times \mathbf{B})+\mathbf{B} \times(\boldsymbol{\nabla} \times \mathbf{A}) .
\end{aligned}
$$

Setting $\mathbf{A}=\nabla p$ and $\mathbf{B}=\nabla p^{*}$ causes the curls on the righthand side of Eq. (A6) to vanish identically. The two remaining terms yield

$$
\begin{aligned}
\operatorname{Re}\left\{(\nabla p \cdot \nabla) \nabla p^{*}\right\} & =\frac{1}{2} \nabla\left(\nabla p \cdot \nabla p^{*}\right) \\
& =\frac{1}{2} \nabla\left[(\nabla u)^{2}+u^{2}(\nabla \varphi)^{2}\right] .
\end{aligned}
$$

Combining this with Eq. (A2a) and the identity $\nabla^{2}\left(u^{2}\right)=$ $2 u \nabla^{2} u+2(\nabla u)^{2}$ yields Eq. (9b).

Equation (9c) similarly follows from the identity

$$
\begin{aligned}
\nabla \times(\mathbf{A} \times \mathbf{B})= & \mathbf{A}(\nabla \cdot \mathbf{B})-\mathbf{B}(\nabla \cdot \mathbf{A}) \\
& +(\mathbf{B} \cdot \nabla) \mathbf{A}-(\mathbf{A} \cdot \nabla) \mathbf{B} .
\end{aligned}
$$

Setting $\mathbf{A}=\nabla p$ and $\mathbf{B}=\nabla p^{*}$, we obtain

$$
\operatorname{Im}\left\{(\nabla p \cdot \nabla) \nabla p^{*}\right\}=-\operatorname{Im}\left\{\nabla^{2} p \nabla p^{*}\right\}+\frac{i}{2} \nabla \times\left(\nabla p \times \nabla p^{*}\right) .
$$

We use the Helmholtz equation to eliminate the Laplacian operator on the right-hand side so that

$$
\begin{aligned}
\operatorname{Im}\left\{\nabla^{2} p \nabla p^{*}\right\} & =k^{2} \operatorname{Im}\left\{p \nabla p^{*}\right\} \\
& =-k^{2} u^{2} \nabla \varphi
\end{aligned}
$$


with Eq. (A12) following from Eq. (A4). As before, this term is divergence free and therefore corresponds to a purely nonconservative force.

The second term on the right-hand side of Eq. (A10) is the curl of a function and thus also corresponds to a nonconservative force. It may be expressed in terms of the wave's amplitude and phase profiles as

$$
\begin{aligned}
\frac{i}{2} \nabla & \times\left(\nabla p \times \nabla p^{*}\right) \\
& =\nabla \times[u(\nabla u \times \nabla \varphi)] \\
& =u \nabla \times(\nabla u \times \nabla \varphi)+\nabla u \times(\nabla u \times \nabla \varphi) .
\end{aligned}
$$

The first term on the right-hand side of Eq. (A13) can be rewritten as

$$
\begin{aligned}
u \nabla \times(\nabla u \times \nabla \varphi)= & u\left(\nabla^{2} \varphi+\nabla \varphi \cdot \nabla\right) \nabla u \\
& -u\left(\nabla^{2} u+\nabla u \cdot \nabla\right) \nabla \varphi,
\end{aligned}
$$

while the second can be expressed as

$$
\nabla u \times(\nabla u \times \nabla \varphi)=(\nabla u \cdot \nabla \varphi) \nabla u-(\nabla u \cdot \nabla u) \nabla \varphi .
$$

Using Eq. (A2b) to eliminate $\nabla u \cdot \nabla \varphi$ and combining all remaining terms yields Eq. (9c).
[1] L. Zhang and P. L. Marston, Angular momentum flux of nonparaxial acoustic vortex beams and torques on axisymmetric objects, Phys. Rev. E 84, 065601(R) (2011).

[2] W. Wang, L. A. Castro, M. Hoyos, and T. E. Mallouk, Autonomous motion of metallic microrods propelled by ultrasound, ACS Nano 6, 6122 (2012).

[3] A. Marzo, S. A. Seah, B. W. Drinkwater, D. R. Sahoo, B. Long, and S. Subramanian, Holographic acoustic elements for manipulation of levitated objects, Nat. Commun. 6, 8661 (2015).

[4] A. Marzo and B. W. Drinkwater, Holographic acoustic tweezers, Proc. Natl. Acad. Sci. USA 116, 84 (2019).

[5] W. J. Xie, C. D. Cao, Y. J. Lü, Z. Y. Hong, and B. Wei, Acoustic method for levitation of small living animals, Appl. Phys. Lett. 89, 214102 (2006).

[6] H.-L. Cao, D.-C. Yin, Y.-Z. Guo, X.-L. Ma, J. He, W.-H. Guo, X.-Z. Xie, and B.-R. Zhou, Rapid crystallization from acoustically levitated droplets, J. Acoust. Soc. Am. 131, 3164 (2012).

[7] M. A. B. Andrade, A. L. Bernassau, and J. C. Adamowski, Acoustic levitation of a large solid sphere, Appl. Phys. Lett. 109, 044101 (2016).

[8] A. Lenshof, C. Magnusson, and T. Laurell, Acoustofluidics 8: Applications of acoustophoresis in continuous flow microsystems, Lab Chip 12, 1210 (2012).

[9] C. R. P. Courtney, B. W. Drinkwater, C. E. M. Demore, S. Cochran, A. Grinenko, and P. D. Wilcox, Dexterous manipulation of microparticles using Bessel-function acoustic pressure fields, Appl. Phys. Lett. 102, 123508 (2013).

[10] V. Bjerknes, Fields of Force (Columbia University Press, New York, 1906).

[11] F. G. Blake, Jr., Bjerknes forces in stationary sound fields, J. Acoust. Soc. Am. 21, 551 (1949).

[12] L. P. Gor'kov, On the forces acting on a small particle in an acoustical field in an ideal fluid, Sov. Phys. Dokl. 6, 773 (1962).

[13] M. Settnes and H. Bruus, Forces acting on a small particle in an acoustical field in a viscous fluid, Phys. Rev. E 85, 016327 (2012).

[14] G. T. Silva, Acoustic radiation force and torque on an absorbing compressible particle in an inviscid fluid, J. Acoust. Soc. Am. 136, 2405 (2014).

[15] J. P. Gordon, Radiation forces and momenta in dielectric media, Phys. Rev. A 8, 14 (1973).
[16] P. C. Chaumet and M. Nieto-Vesperinas, Time-averaged total force on a dipolar sphere in an electromagnetic field, Opt. Lett. 25, 1065 (2000).

[17] D. B. Ruffner and D. G. Grier, Comment on Scattering Forces from the Curl of the Spin Angular Momentum of a Light Field, Phys. Rev. Lett. 111, 059301 (2013).

[18] A. Yevick, D. B. Ruffner, and D. G. Grier, Tractor beams in the Rayleigh limit, Phys. Rev. A 93, 043807 (2016).

[19] A. Yevick, D. J. Evans, and D. G. Grier, Photokinetic analysis of the forces and torques exerted by optical tweezers carrying angular momentum, Phil. Trans. R. Soc. A 375, 20150432 (2017).

[20] A. Ashkin, J. M. Dziedzic, J. E. Bjorkholm, and S. Chu, Observation of a single-beam gradient force optical trap for dielectric particles, Opt. Lett. 11, 288 (1986).

[21] P. Y. Wu, R. X. Huang, C. Tischer, A. Jonas, and E. L. Florin, Direct Measurement of the Nonconservative Force Field Generated by Optical Tweezers, Phys. Rev. Lett. 103, 108101 (2009).

[22] Y. Roichman, B. Sun, Y. Roichman, J. Amato-Grill, and D. G. Grier, Optical Forces Arising from Phase Gradients, Phys. Rev. Lett. 100, 013602 (2008).

[23] B. Sun, J. Lin, E. Darby, A. Y. Grosberg, and D. G. Grier, Brownian vortexes, Phys. Rev. E 80, 010401(R) (2009).

[24] L. Allen, M. W. Beijersbergen, R. J. C. Spreeuw, and J. P. Woerdman, Orbital angular-momentum of light and the transformation of Laguerre-Gaussian laser modes, Phys. Rev. A 45, 8185 (1992).

[25] H. He, M. E. J. Friese, N. R. Heckenberg, and H. RubinszteinDunlop, Direct Observation of Transfer of Angular Momentum to Absorptive Particles from a Laser Beam with a Phase Singularity, Phys. Rev. Lett. 75, 826 (1995).

[26] K. T. Gahagan and G. A. Swartzlander, Optical vortex trapping of particles, Opt. Lett. 21, 827 (1996).

[27] N. B. Simpson, L. Allen, and M. J. Padgett, Optical tweezers and optical spanners with Laguerre-Gaussian modes, J. Mod. Opt. 43, 2485 (1996).

[28] K. Ladavac, K. Kasza, and D. G. Grier, Sorting by periodic potential energy landscapes: Optical fractionation, Phys. Rev. E 70, 010901(R) (2004).

[29] K. Ladavac and D. G. Grier, Colloidal hydrodynamic coupling in concentric optical vortices, Europhys. Lett. 70, 548 (2005). 
[30] S.-H. Lee, Y. Roichman, and D. G. Grier, Optical solenoid beams, Opt. Express 18, 6988 (2010).

[31] B. T. Draine, The discrete-dipole approximation and its application to interstellar graphite grains, Astrophys. J. 333, 848 (1988).

[32] S. Albaladejo, R. Gómez-Medina, L. S. Froufe-Pérez, H. Marinchio, R. Carminati, J. F. Torrado, G. Armelles, A. GarcíaMartin, and J. J. Sáenz, Radiative corrections to the polarizability tensor of an electrically small anisotropic dielectric particle, Opt. Express 18, 3556 (2010).

[33] O. A. Sapozhnikov and M. R. Bailey, Radiation force of an arbitrary acoustic beam on an elastic sphere in a fluid, J. Acoust. Soc. Am. 133, 661 (2013).

[34] M. X. Lim, K. A. Murphy, and H. M. Jaeger, Edges control clustering in levitated granular matter, Granul. Matter 21, 77 (2019).

[35] H. E. Bass, L. C. Sutherland, A. J. Zuckerwar, D. T. Blackstock, and D. M. Hester, Atmospheric absorption of sound: Further developments, J. Acoust. Soc. Am. 97, 680 (1995).

[36] M. A. Ainslie and J. G. McColm, A simplified formula for viscous and chemical absorption in sea water, J. Acoust. Soc. Am. 103, 1671 (1998).

[37] J. Lighthill, Acoustic streaming, J. Sound Vibration 61, 391 (1978).
[38] I. D. Toftul, K. Y. Bliokh, M. I. Petrov, and F. Nori, Acoustic Radiation Force and Torque on Small Particles as Measures of the Canonical Momentum and Spin Densities, Phys. Rev. Lett. 123, 183901 (2019).

[39] D. Bazou, A. Castro, and M. Hoyos, Controlled cell aggregation in a pulsed acoustic field, Ultrasonics 52, 842 (2012).

[40] K. Melde, A. G. Mark, T. Qiu, and P. Fischer, Holograms for acoustics, Nature (London) 537, 518 (2016).

[41] C. E. M. Démoré, P. M. Dahl, Z. Yang, P. Glynne-Jones, A. Melzer, S. Cochran, M. P. MacDonald, and G. C. Spalding, Acoustic Tractor Beam, Phys. Rev. Lett. 112, 174302 (2014).

[42] J. Masajada and B. Dubik, Optical vortex generation by three plane wave interference, Opt. Commun. 198, 21 (2001).

[43] K. Y. Bliokh and F. Nori, Spin and orbital angular momenta of acoustic beams, Phys. Rev. B 99, 174310 (2019).

[44] P. L. Marston, Axial radiation force of a Bessel beam on a sphere and direction reversal of the force, J. Acoust. Soc. Am. 120, 3518 (2006).

[45] J. Durnin, Exact-solutions for nondiffracting beams. 1. The scalar theory, J. Opt. Soc. Am. A 4, 651 (1987).

[46] J. Durnin, J. J. Miceli, Jr., and J. H. Eberly, Diffraction-free beams, Phys. Rev. Lett. 58, 1499 (1987).

[47] X.-D. Fan and L. Zhang, Trapping Force of Acoustical Bessel Beams on a Sphere and Stable Tractor Beams, Phys. Rev. Appl. 11, 014055 (2019). 